\title{
Determining and interpreting correlations in lipidomic networks found in glioblastoma cells
}

\author{
Robert Görke ${ }^{1 *}$, Anke Meyer-Bäse ${ }^{2^{*}}$, Dorothea Wagner ${ }^{1}$, Huan He ${ }^{3}$, Mark R Emmett ${ }^{3,4}$, Charles A Conrad ${ }^{5}$
}

\begin{abstract}
Background: Intelligent and multitiered quantitative analysis of biological systems rapidly evolves to a key technique in studying biomolecular cancer aspects. Newly emerging advances in both measurement as well as bio-inspired computational techniques have facilitated the development of lipidomics technologies and offer an excellent opportunity to understand regulation at the molecular level in many diseases.

Results: We present computational approaches to study the response of glioblastoma U87 cells to gene- and chemo-therapy. To identify distinct biomarkers and differences in therapeutic outcomes, we develop a novel technique based on graph-clustering. This technique facilitates the exploration and visualization of co-regulations in glioblastoma lipid profiling data. We investigate the changes in the correlation networks for different therapies and study the success of novel gene therapies targeting aggressive glioblastoma.

Conclusions: The novel computational paradigm provides unique "fingerprints" by revealing the intricate interactions at the lipidome level in glioblastoma U87 cells with induced apoptosis (programmed cell death) and thus opens a new window to biomedical frontiers.
\end{abstract}

\section{Background}

Glioblastoma are highly invasive brain tumors. The prognosis for patients with glioblastoma depends on many factors, including age, performance status, and histology grade of the glial neoplasm. The medial survival is approximately 14 months with maximal therapy. Glioblastoma are difficult to treat due to the resistance to conventional therapies as well as the ability to diffuse throughout the brain. Therefore, analytical description of tumor growth and response to therapeutic modalities, such as radiation and chemotherapy, has been a central research topic. Modeling of the tumor responding to chemotherapy is mostly of pharmacokinetic nature [1]. Other mathematical models are based on a conservation equation describing a modality of how to measure the growth of an infiltrating glioma [2]: the rate of change of tumor cell population equals the diffusion (motility) of tumor cells plus the net proliferation of those. Cell death is introduced as a loss term in [3]. A model

\footnotetext{
* Correspondence: robert.goerke@kit.edu; ameyerbaese@fsu.edu ${ }^{1}$ Department of Computer Science, Karlsruhe Institute of Technology, Karlsruhe D-76128, Germany

${ }^{2}$ Department of Scientific Computing, Florida State University, Tallahassee, Florida 32310-4120, USA

Full list of author information is available at the end of the article
}

combining patient's imaging, histopathologic and pharmacodynamic/genetic data when treated with temozolomide is presented in [4].

Interesting aspects of glial cell biology have recently been uncovered in laboratories evaluating the tumor suppressor protein wild type 53 (wt p53) [5,6]. It is well established that transfecting glioma cells with wild-type tumor protein p53 will trigger brisk apoptosis if the cell line harbors mutant $\mathrm{p} 53$, while the same transfection to cell lines which harbor the wt p53 will result in a reduction or elimination of invasion and motility [7]. A glioma cell line that harbors the wild-type form of the tumor suppressor protein p53 can be sensitized to undergo apoptosis by the addition of wt p53 along with chemotherapy (such as SN38) [5,6]. Recently, new insights into the pathobiology of glioblastoma cells have been obtained at the M.D. Anderson Cancer Center in Houston, Texas: transfer of the p53 gene by use of an adenovirus vector (Ad-p53) may be clinically applicable in human gliomas. Furthermore, it has been demonstrated that combined adenovirus transfection of wild-type p53 (wt p53) into glioma cells followed with chemotherapy treatment SN-38 may act to convert gliomas to an "apoptosis-sensitive" phenotype [8]. Moreover, wt p53 containing tumor cells, such as U87 
MG will show reduced mobility and decreased invasion in matrigel motility assays after wt p53 gene therapy. A proteomic approach identified proteins that were involved in a phenotypic change in the high-grade glioma cell line U87 MG under the influence of transfection with wildtype p53 and additional cytotoxic chemotherapy with SN-38 [6]. This study showed that the expression of the protein galectin-1 is associated with malignancy and poor prognosis. The results suggest that galectin-1 is a relevant therapeutic target to downregulate in a clinical pharmacological setting to improve overall survival of high-grade glioma patients. Our current understanding of proteins such as galectin-1, interactions and pathways is detailed, yet it is still incomplete. Galectin 1 binds free beta-galactose residues on both glycoproteins and gangliosides (GM1 and asialo-GM1 gangliosides are known to be galectin-1 ligands) [9]. Gangliosides were first discovered by the German scientist Ernst Klenk in 1942. They are cell-type specific antigens that provide cell membrane structure, and play key roles in control growth, cell differentiation and cell/cell interactions. Gangliosides are implemented in different cancer types (such as glioblastoma) since some typical gangliosides are present in tumors, but are absent in normal healthy tissue [10].

Although connections between cancer and glycobiology have been described, the detailed chemical analysis of polar lipids has been problematic due to structural complexity as well as limitation of analytical techniques. Recently, He et al. [11] pioneered new analytical methodology with nano-liquid chromatography (nano-LC) separation followed by high mass accuracy and high mass-resolving power Fourier transform ion cyclotron resonance (FT-ICR) mass spectrometry (MS) analysis at 14.5T [12]. MS is useful in dealing with complex mixtures since the high mass resolution (narrow peak width) allows the signals of two ions of similar mass-to-charge ratio $(\mathrm{m} / \mathrm{z})$ to be detected as distinct ions. This new methodology has opened a new field of polar lipid profiling. A typical biological analysis from 2 million cells results in the identification of 600-800 different polar lipids of various polar heads and variable size and modified (degree of saturation and hydroxylation) nonpolar tails. Relative quantification of each polar lipid between treated and untreated cells results in highly complex, information rich data sets. The ultimate goal of this research is to link the changes in the polar lipids to the enzymes that control their synthesis/modifications/degradation (gene arrays and functional proteomics), which will provide a systems biology approach to the study of glioblastoma. The data generated will be used to drive logical, hypothesis driven experiments in the search for new therapeutic targets in glioblastoma research.

Since the lipid profiling methodology generates comparative semi-quantitative data on hundreds of different polar lipids with each experiment, the data analysis has become a daunting task. Novel approaches [13-15] are now needed to compile the existing biological lipid profiling experimental data and to maximize the information extraction from these databases. Once correlations in the lipid database have been identified, the reduced data will be correlated to the gene array data and proteomic data sets. This manuscript describes a novel algorithm for identification of correlations in the complex lipid data sets derived from gene treated and empty vector treated U87 glioblastoma cell lines. We research, implement and test graph clustering techniques as an equivalent to standard correlation networks. This novel approach combines analysis and visualization with the correlation structure of the lipid profiling data. This novel technique enables a better understanding and a mathematical analysis of the observed experimental results.

\section{Results and Discussion}

In this paper we develop a novel paradigm to replace correlation networks standardly employed for displaying the topological properties of networks $[16,17]$. Traditional correlation networks are based on building a graph and determining the correlation scores between the nodes of the graph and then compare those values with a given threshold. For those exceeding the threshold an undirected edge is linked between the nodes. As a novel concept in this paper, we research, implement and test graph clustering techniques as an equivalent to standard correlation networks for the analysis of therapeutic outcomes for U87 MG glioblastoma cells.

Global multivariate approaches such as the proposed novel graph clustering correlation network are needed in order to understand the potential disregulation of normal cellular responses in disease and their response to various therapeutic interventions. Moreover, because nano-LC/MS can find and assign hundred of lipids from glioma cells, it is possible to identify causal influence relationships involving multiple lipids. Building upon lipid measurements such as those illustrated in Figure 1 for the group of gangliosides for two treatments (here, treatments one and two listed in Table 1), Figure 2 shows the schematic of the graph clustering network based on multidimensional lipidomic data. The correlation structure is explored by varying the threshold between different lipids and the important changes between treatments by clusters of nodes of different sizes and fan-out degrees.

\section{A Graph Model based on Correlation Networks}

The notion of networks has recently started to permeate diverse fields of science as well as numerous concepts in our everyday lives. This development roots 


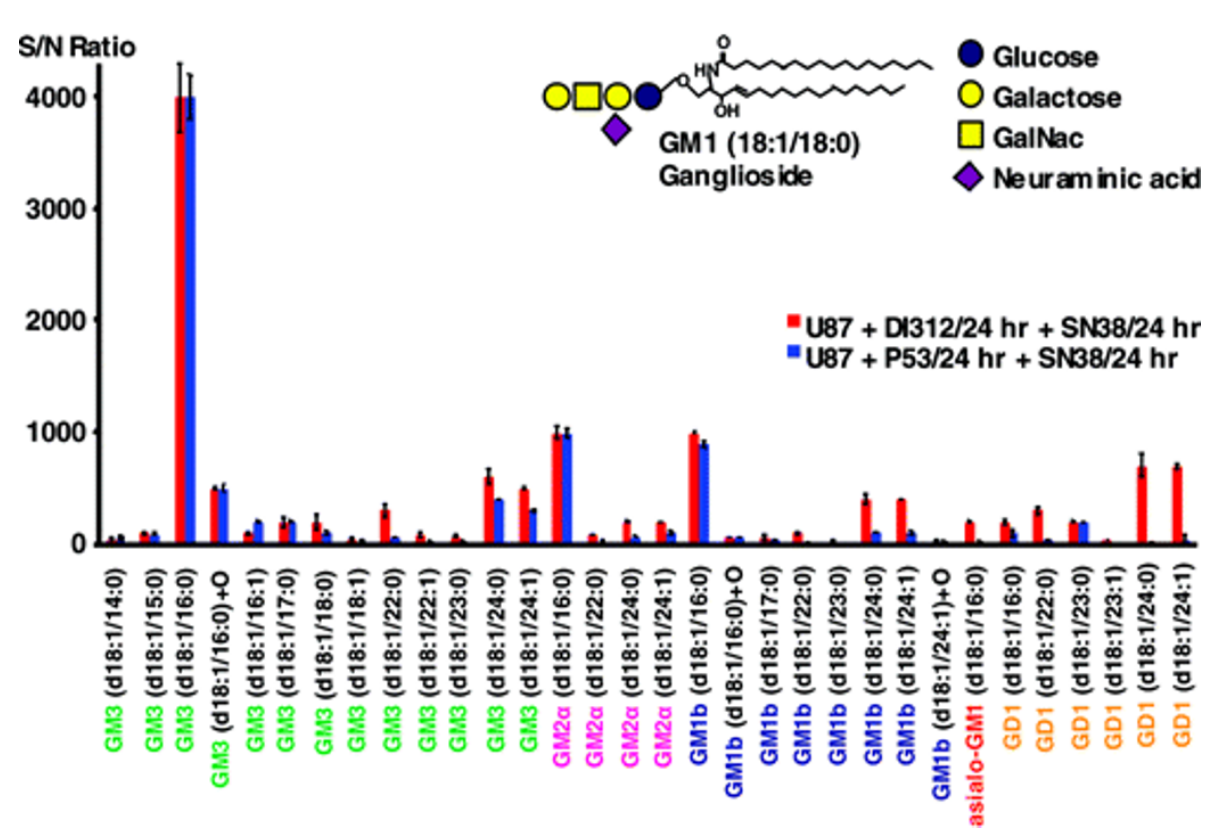

Figure 1 Gangliosides profiles for the two treatments U87+DI312/24hr+SN38/24hr and U87+p53/24hr+SN38/24hr. Ganglioside profiles for the polar lipid fraction of extracts of U87 cells following two different treatments. The effect of treatment with wild-type wt p53, which lowers the abundance of longer chain ceramides, is most pronounced for the GM1b and GD1 gangliosides. Figure extracted from [11] and awaiting copyright permission.

particularly strong in social network analysis, an area which spawned many concepts and methods that today experience a renaissance empowered by the ubiquity and availability of social networks, especially in the internet (another, technical network). The driving force behind the rise of this concept is the fact that a network is a very apt and well-perceivable representation of the interrelation of things. For this reason, we advocate the use of network analysis [18] in the field of lipidomics. Generally speaking, any set of interrelated entities constitutes a network, and can thus be modeled as a graph, which is the mathematical formalization of the term network. The evidence that lipids

Table 1 Treatment conditions of U87 Glioblastoma Cell Cultures.

\begin{tabular}{|c|c|}
\hline \multicolumn{2}{|c|}{ Treatment conditions of U87 Glioblastoma Cell Cultures } \\
\hline Condition & Abbreviation \\
\hline Treatment 1 & U87 + DI312/24h + SN38/24h \\
\hline Treatment 2 & $\mathrm{U} 87+\mathrm{p} 53 / 24 \mathrm{~h}+\mathrm{SN} 38 / 24 \mathrm{~h}$ \\
\hline Treatment 3 & $\mathrm{U} 87+\mathrm{SN} 38 / 24 \mathrm{~h}+\mathrm{DI} 312 / 24 \mathrm{~h}$ \\
\hline Treatment 4 & $\mathrm{U} 87+\mathrm{SN} 38 / 24 h+\mathrm{p} 53 / 24 h$ \\
\hline Treatment 5 & $\mathrm{U} 87+\mathrm{D} 1312$ \\
\hline Treatment 6 & $\mathrm{U} 87+\mathrm{p} 53 / 24 \mathrm{~h}$ \\
\hline Treatment 7 & U87 + SN38/24h \\
\hline Control & U87 cell only \\
\hline
\end{tabular}

are related in their reaction to treatments leads to the fact that exploration and visualization of co-regulations in glioblastoma data can be done based on correlation network analysis. Such networks describe all dependencies between the domain variables. More precisely we define the simple (neither self-loops nor parallel edges are allowed), undirected (edges represent a mutual relationship) and weighted graph $G=(V, E, \omega)$ as follows. The set $V$ of nodes is the set of all measured lipids while the set $E$ of edges (i.e nodes pairs) describes the dependencies between these lipids by a weight function $\omega: E \rightarrow[0,1]$ which expresses the strength of the dependencies. In our case $\omega$ is based on a matrix of correlation coefficients which is computed based on the pair-wise correlation between the concentrations of lipids in a given sample. Thus, for an edge $e=\{u, v\}$, the weight $\omega(e)$ is a measure of correlation, i.e., similarity between lipids $u$ and $v$, with a value of 1 indicating identical measurements $x$ and $y$, and values close to 0 indicating very different measurements. We base our correlation matrix on measured values $x$ normalized with the standard normalization function $f(x)=\log (x+1)$. However, since this proved to be too non-discriminative for network analysis techniques, we actually used $f^{\prime}(x)=(f(x))^{3}$. The correlation of two measurements $x$ and $y$ is then computed as $\operatorname{cor}(x$, $y)=\min \left\{f^{\prime}(x), f^{\prime}(y)\right\} / \max \left\{f^{\prime}(x), f^{\prime}(y)\right\}$, with the special case of $\operatorname{cor}(x, y)=1$ if $x=y=0$. 


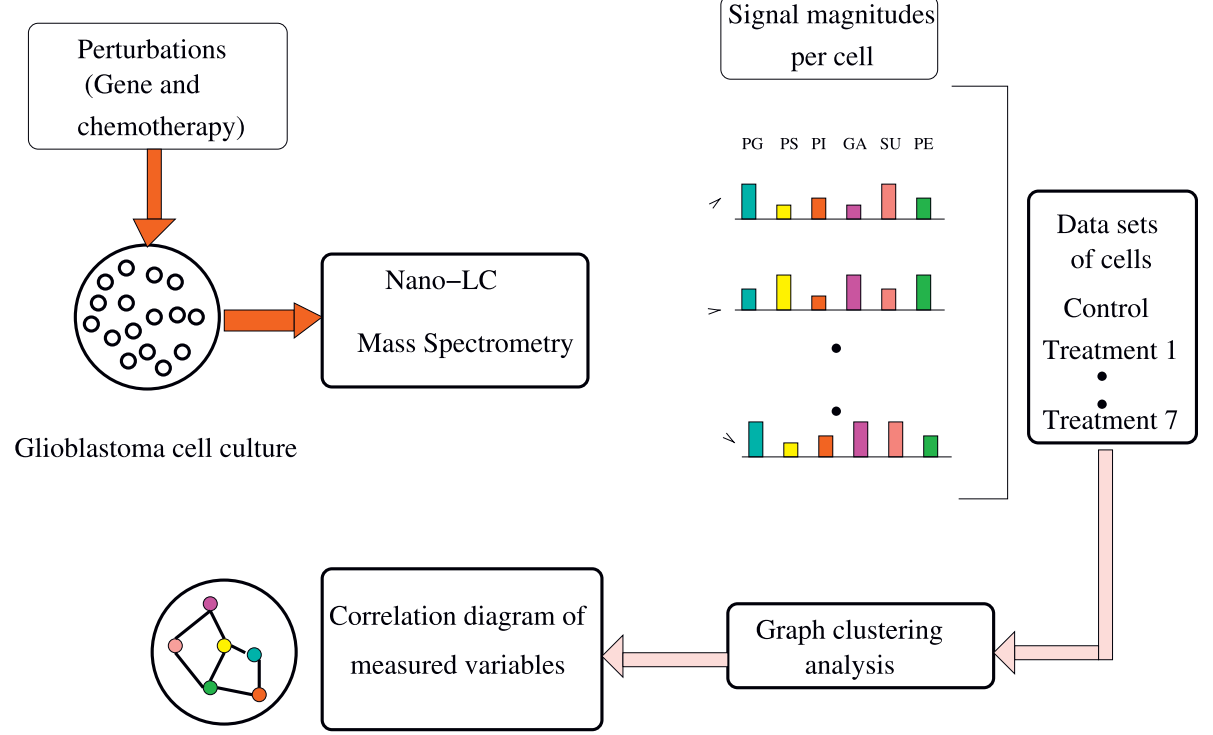

Figure 2 Lipidomic platform data processing. Graph clustering modeling with U87 MG glioblastoma cell data. The schematic shows a resulting correlation based on nano-LC/MS data. Seven different treatments are applied to a cell line. Graph clustering analysis is performed on this data and a correlation diagram reflecting dependencies in the underlying network is obtained. Abbreviations. PG means

phosphatidylglycerol, PS means phosphatidylserine, PI means phosphatidylinositol, PE means phosphatidylethanolamine, GA means gangliosides and SU means sulfatide.

Since in this work we divide our studies up into different groups of lipids, i.e., gangliosides, phosphatidylinositol and phosphatidylglycerol, we construct three different base-networks for our exploratory clusterings, one for each group. Actual lipid correlation graphs are then obtained by choosing a treatment and a threshold value for the correlation coefficient values, i.e., edges with insignificant weights are pruned. Two nodes $u$ and $v$ that are connected by an edge are called adjacent, and an edge $e$ and an end node $v$ of $e$ are called incident, abbreviated by $u \sim v$ and $e \sim v$, respectively. Figure 3 is the network of phosphatidylglycerols. For the sake of readability we do not state full names of lipids in our graphs, but instead denote the index of the lipid in the corresponding chart (as, e.g., in Figure 3 for phosphatidylglycerols). The layout (i.e., the positions of nodes in a drawing) is determined with so called force-directed graph-drawing techniques. Roughly speaking, strong ties draw nodes together and weak ties let them drift apart. Heavier edges are darker than lighter edges and larger nodes share many heavy edges with other nodes. We can now identify groups of lipids that exhibit a similar behavior.

\section{Graph Clustering on Correlation Networks}

We propose and tested a novel concept for analyzing correlation networks based on graph clustering. In Figure 3 we can easily perceive three rather densely knit groups of lipids, which share mostly weak links (if at all) to the other groups. Such a dense subset indicates that these lipids correlate strongly with each other wrt. to their reaction to the applied treatment, i.e., it is a cluster of similarly behaving lipids. While in a single small example this might be done by hand, this task is usually realized with graph clustering $[18,19]$ algorithms, which automatically identify a clustering. Clustering methods discover natural concentration and large-scale inhomogeneities in relational data. A clustering $\mathcal{C}(G)$ of a graph $G=(V, E)$ is a partition $\mathcal{C}=\left\{C_{1}, \ldots, C_{k}\right\}$ of the set $V$ of nodes into clusters $C_{i}$ (remember that a partition $\mathscr{B},=\left\{B_{1}, \ldots, B_{k}\right\}$ of a set $A$ always guarantees $\bigcup_{i=1}^{k} B_{i}=A$ and $\left.B_{i} \cap B_{j}=\varnothing, \forall i \neq j \in\{1, \ldots, k\}\right)$.

Clusterings always follow the paradigm of intra-cluster density vs. inter-cluster sparsity. In other words, the edge-connectivity inside clusters is to be strong, while between clusters there should only be few, or lightweighted edges. In our context this means that the pairwise correlations of nodes inside one cluster are all rather high, and those of nodes in different clusters are comparably low. It is important to note that the number of clusters to be found is not preset by a parameter, instead it is determined by the algorithm and the instance. Since edge weights represent the strength of ties, our graph clustering algorithm takes these edge weights into account, which yields a higher precision than pruning light edges and then employing an un weighted algorithm. For the sake of readability, we still use the pruning only for our drawings. 


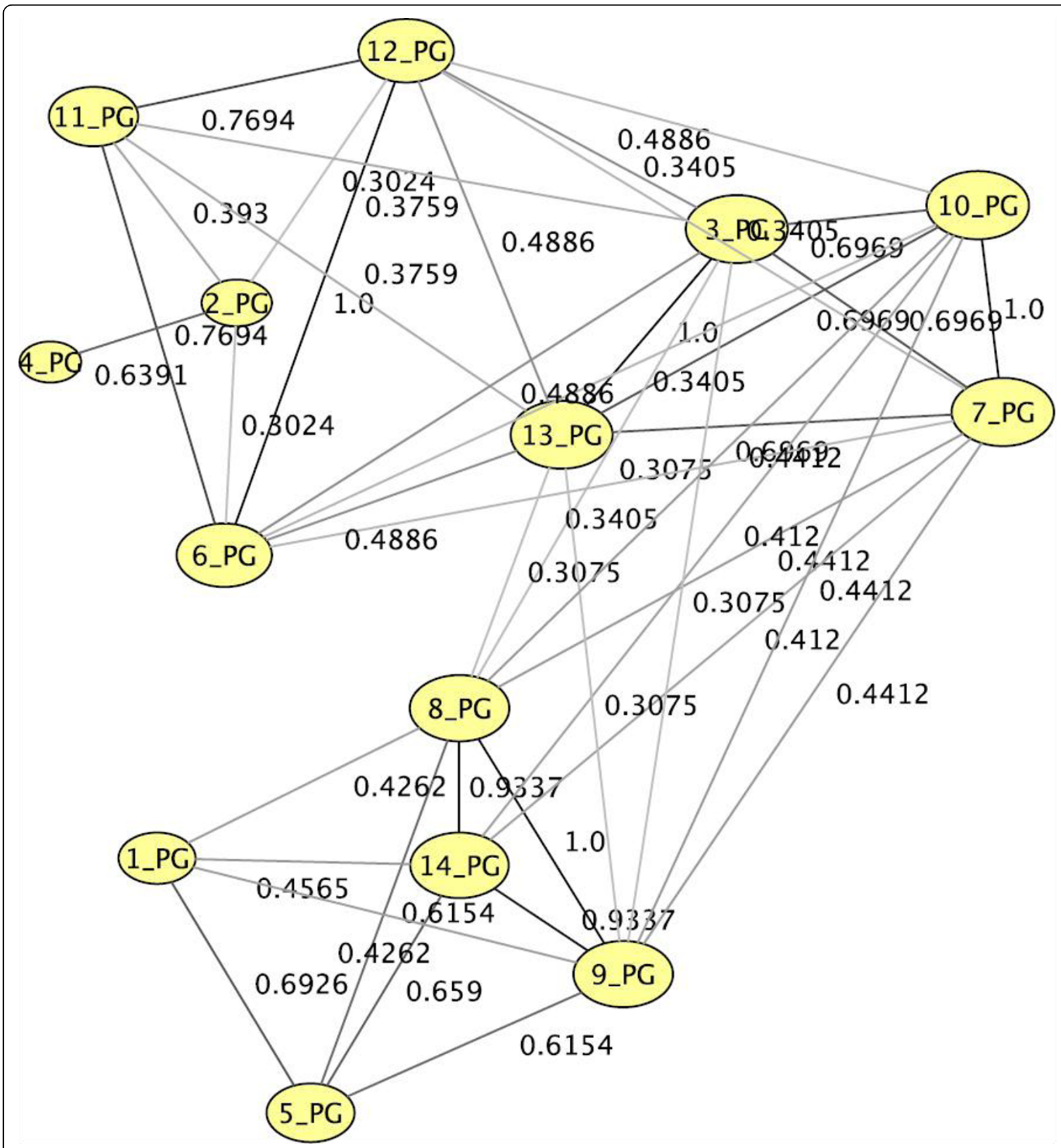

Figure 3 Example of a correlation network for phosphatidylglycerols for U87+SN38/24hr. A correlation network of the group of phosphatidylglycerols wrt. treatment 7 (see Tab. 1), based on a threshold of 0.3.

For actual clustering tasks, the informal notion of intra-cluster density vs. inter-cluster sparsity requires amathematical formalization. A very simple such formula is coverage, which measures the fraction of thetotal edge weight which is covered by (i.e., inside) clusters (see Formula 1). While, for a given clustering, coverage is a simple, yet decent measure for clustering goodness, trying to maximize it is unreasonable, as an optimal coverage value of 1 is attained by setting $\mathcal{C}=\left\{C_{1}\right\}$ with $C$ $=V$, i.e., using only one cluster. Among the established graph clustering algorithms that have successfully been applied to networks with similar structure in the past are algorithms that maximize the quality index modularity [20], which builds upon coverage. Roughly speaking, 
this index measures the goodness of a clustering (on a scale from -1 to 1 ) by comparing the coverage of a clustering to the expectation of this value if edges were rewired randomly. Equations (1) and (2) give the formulas for coverage and modularity, respectively:

$$
\begin{aligned}
& \operatorname{cov}(\mathcal{C}):=\frac{\text { intra } \square \text { cluster edges } e^{\omega(e)}}{\sum_{e \in E} \omega(e)} \\
& \bmod (\mathcal{C}):=\operatorname{cov}(\mathcal{C})-\underbrace{4\left(\sum_{e \in E} \omega(e)\right)^{2}}_{\text {Exp[coverage }]} \sum_{C \in \mathcal{C}}\left(\sum_{v \in \mathcal{C}} \sum_{e \sim v} \omega(e)\right)^{2}
\end{aligned}
$$

For the purpose of this article it is unnecessary to delve into the details of modularity, we refer the reader to [21-23] and further references therein. The rationale behind modularity is as follows. Given some clustering, a very low modularity tells us that even a purely random network would $t$ the clustering better than the given graph, while a high modularity score means that the identified clustering captures very well how edges differ from randomness and constitute dense groups. The modularity of given clustering can be computed in linear time (linear in $|\mathrm{V}|+|\mathrm{E}|$ ), however, identifying a clustering with a high modularity is harder. In fact it is NP-hard to actually optimize modularity [21], thus heuristics are used, of which a localized agglomerative approach [24] is the current state of the art, which we also use in this work. (The term "NP-hard" from theoretical computer science indicates that it is highly unlikely that an algorithm with a practicable-subexponential in the size of the input-running time for large instances of this problem exists, roughly speaking, a heuristic is a strategy which does a good job, but no qualitative guarantee about its behavior can be given a priori.) We repeated our experiments with other graph clustering algorithms such as ORCA[25], which is strong on very large graphs, minimum-cut tree clustering [26], which is able to guarantee certain bottleneck properties of clusters, and iterative conductance clustering [27], which features a parameterized clustering coarseness. However, the constructed graphs are not large and we do not impose any specific requirements on the clustering, instead we intend to learn from the clustering and get an impression of the network. For these reasons, a selfcontained modularity-based algorithm worked best for our purpose.

The algorithm we use [21] initializes each node as a singleton cluster, i.e., containing only this one node. Then, iteratively, each node explores its local graph neighborhood, leaves its current cluster and joins the best neighbor cluster, if such a swap yields an increase in modularity. This process usually merges many clusters. The iteration is repeated until annealed, which means that modularity can no further be improved by moving any single node to a different cluster. Resulting clusters are then abstracted to super-nodes, which means that a new graph is constructed, where each node represents a cluster in the original graph, and edges between original nodes are summarized into super-edges in the abstracted graph. In this resulting graph, the above process is repeated. Eventually, at some abstraction stage, modularity will attain its peak and no node will join any other's cluster, then the abstraction hierarchy is unfurled and the final supernodes induce the clustering of the original graph. Figure 4 is an example clustering of treatment 6 of the phosphatidylglycerols.

\section{Difference Graphs and Visualization of Lipidomic Changes} For each treatment (see Table 1) and for each group of lipids we now design a correlation network as described in the previous section, and identify a graph clustering. Each clustering reveals subsets (clusters) of lipids which exhibit a very similar reaction to this treatment. Thus, roughly speaking, lipids within the same cluster can be grouped in terms of their behavior and, on the other hand, are more or less well separated from lipids in other clusters in this respect.

Generally speaking, comparing a treatment to others, e.g., a control, is of particular interest. Thus, in order to compare a first treatment $a$ to a second treatment $b$ of the same group of lipids, we combine two graphs and their clusterings in one new graph layout, the difference graph, as follows. a's clustering is represented by boxes, such that each box constitutes a cluster, and $b$ 's clustering by node colors, i.e., subsets of nodes with the same color constitute clusters. Comparing these clusterings yields many insights, which are immediately observable in a readable layout. It shows us how differently the two treatments "correlated the lipids". A cluster of lipids that react almost identically wrt. $a$ might be split into different clusters in $b$ 's clustering, which strongly indicates that the split apart nodes (i.e., lipids) share a property that makes them sensitive to treatment $b$. This selectively leads us back to specific places in the raw data, where we now know what to look for. In particular, this might even point to some biochemical feature of these lipids, the relevance of which has not yet been known. We will demonstrate this in the illustration below.

As a side note, there is some literature on quantitatively comparing clusterings of graphs or partitions of data points. A good overview of this field, with an 


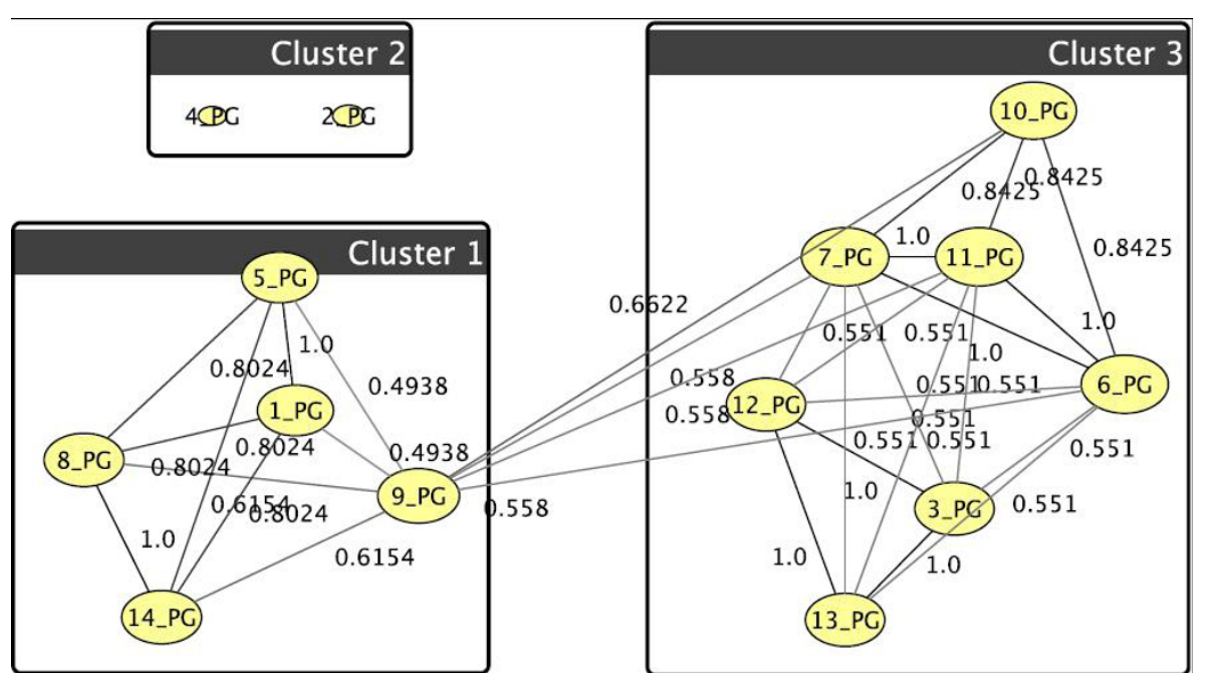

Figure 4 Example of graph-clustering correlation network for phosphatidylglycerols for U87+p53/24hr. The clustered phosphatidylglycerols wrt. treatment 6 (threshold of 0.5). Cluster 1 can also be observed in Fig. 3, cluster 3 includes most other lipids as they behave more similar than for treatment 7, only nodes $2[(32: 0)+\mathrm{O}]$ and $4[(32: 1)+\mathrm{O}]$ are further distant, their weak tie $(0.25<$ threshold) is pruned visually.

emphasis on the peculiarities of graph clusterings, is given in [28]. A multitude of difference measures for clusterings exists, with perhaps the most prominent one being the Rand measure [29], which is the fraction of node pairs, which are co-classified in the same way by both clusterings, i.e., twice together or twice separated. Technically speaking, for two clusterings $\mathcal{C}_{1}$ and $\mathcal{C}_{2}$ of graph $G=(V, E)$ set $n_{\text {tog. }}=\mid\left\{\{u, v\} \in\left(\begin{array}{c}V \\ 2\end{array}\right) \mid \exists C_{1} \in \mathcal{C}_{1}, C_{2} \in \mathcal{C}_{2}\right.$ such that $u, v$ $\in C_{1}$ and $\left.u, v \in C_{2}\right\} \mid$, and set $n_{\text {sep. }}$ analogously for $u, v$ separated, then $\left.\operatorname{Rand}\left(\mathcal{C}_{1}, \mathcal{C}_{2}\right)=\left(n_{\text {tog. }}+n_{\text {sep. }}\right) / \mid\left(\begin{array}{l}V \\ 2\end{array}\right)\right\} \mid$. Using such a measure of comparison in our application would give a vague impression about how much treatments differ in terms of correlation of lipid reaction without looking at the graphs or knowing how the difference manifests. Using any such measure requires care and experience as they are prone to biases introduced by the size of a network and the coarseness of the clusterings. Since, additionally, we clearly focus on qualitative differences, which, for small graphs such as those in our application, our figures give a good impression about, we do not use difference measures.

Since the edge structure of the two compared graphs are already transferred into clusterings, we have the edges of the new difference graph represent more pressing information. the weight of edge $\{u, v\}$ in a difference graph is its weight wrt. a minus its weight wrt. $b$. The crucial observation is as follows. This weight shows how treatments $a$ and $b$ differ in terms of the behavior of $u$ and $v$; if this weight is close to zero, then switching from $a$ to $b$ does not discriminate $u$ and $v$, if it is positive (negative), then $u$ and $v$ correlate stronger (weaker) wrt. treatment $a$ than wrt. $b$. We color edges on a linear $\mathrm{HSV}$-scale (from blue $=$ cold $=$ small difference to red $=$ hot $=$ large difference) by the absolute value of this new edge weight. Thus, edge colors indicate whether switching between treatment $a$ and $b$ discriminates nodes. However, in order not to overburden the drawing, colors encode only the absolute difference between the $a$ and b's correlations; this hints at where to look for interesting behavior, and detailed quantities can then be read o edge annotations or the raw data. Furthermore, we size nodes proportionally to sum of their incident absolute edge weights. Thus, in a difference-graph for $a$ and $b$, a node is large if it differs strongly in its correlations to other nodes wrt. the two treatments, there should thus be several heavy (i.e., warm-colored) incident edges on such a node. Small nodes roughly preserve their correlation behavior in both treatments.

\section{Illustration of the Approach}

Our approach is illustrated by computing the graphcorrelation networks resulting from gene therapy for gangliosides, phosphatidylinositol and phosphatidylglycerol. In the following we illustrate what can be concluded from the networks constructed from these different groups of lipids, as shown in their respective figures. Please note that we do not predetermine the numbers of clusters, thus different treatments can very well yield different numbers of clusters, this simply reflects that the numbers and sizes of lipids with similar behavior depend on which treatment is used. We exemplarily show the resulting correlation networks 


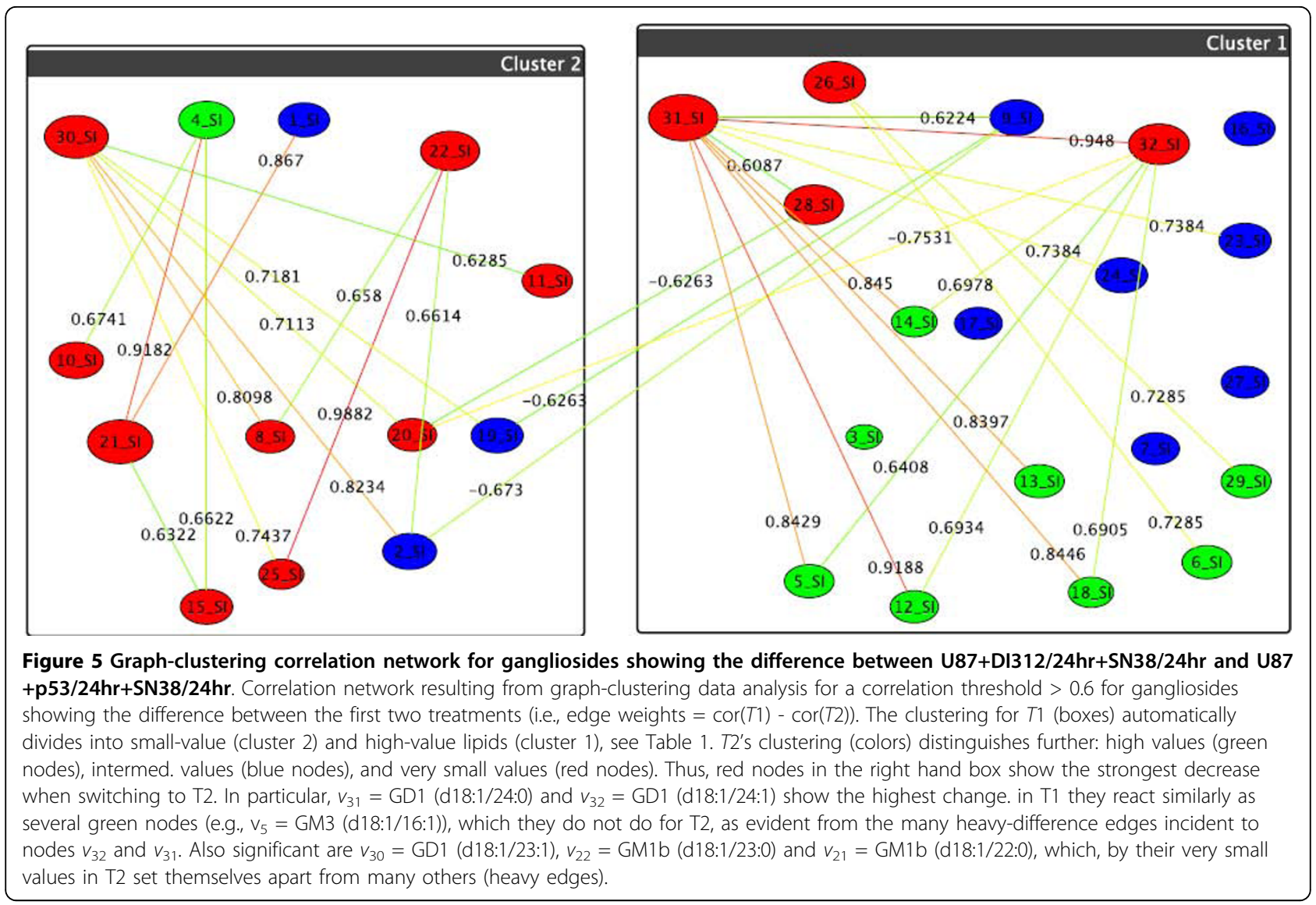

for the group of gangliosides from Figure 1 for the strong difference of the first two treatments from Table 1 and Figure 5. In Figure 5, the result of the control treatment one is summarized by the box-clustering, and the treatment two (p53), is depicted by node colors. A first observation is that the control yields only two clusters of lipids (boxes), and adding p53 yields three (colors), thus, treatment two discriminates the reaction of lipids more finely. The red cluster is striking as it contains many large nodes, i.e., lipids, which differ strongly in their correlations wrt. the two treatments. The fan-out degree of every single lipid component in these graphs shows us the modulation degree with a given treatment. The hot (reddish) edges incident to the largest nodes show where these differences manifest most strongly, i.e., which other nodes are discriminated by such a node (and its cluster) by moving from treatment one to treatment two. Looking up the respective lipids in Figure 1, the graphical clustering thus confirms the experimental results by showing that the long chain gangliosides go down after p53 treatment. In a similar manner we can use our graphs of other groups of lipids and treatments for pointers to behavioral phenomena. For the ratio of the third and fourth treatment combinations and the fifth and sixth, respectively, from Table 1 , we do not observe any relevant changes as shown in Figure 6 and 7.

For the phosphatidylinositol (PI), we see in Figure 8 the experimental outcome for the first two treatments showing an abundance increase of hydroxilated PIs. We can also see that the hydroxylated PI go up after p53 treatment as depicted from the difference network between the first two treatments in Figure 9. The highest increase from zero to one hundred for $(38: 6)+\mathrm{O}$ as shown in the experimental results is represented in the network as a distinct separate cluster. There are no significant changes between the treatments U87+SN38/ $24 \mathrm{hr}+\mathrm{DI} 312 / 24 \mathrm{hr}$ versus $\mathrm{U} 87+\mathrm{SN} 38 / 24 \mathrm{hr}+\mathrm{p} 53 / 24 \mathrm{hr}$ and U87+DI312/24hr versus U87+p53/24hr.

The biochemical observations are again confirmed by our analysis for the phosphatidylglycerol (PG) profiles. The results for the first two treatments only are visualized in Figure 10 showing an abundance increase of hydroxylated PGs. The correlation network confirming these results is shown in Figure 11. Cluster 2 includes only the two PGs $(32: 0)+\mathrm{O}$ and $(32: 1)+\mathrm{O}$ showing an increase in signal magnitude from 0 for the first treatment to 100 for the second one. There are again as 


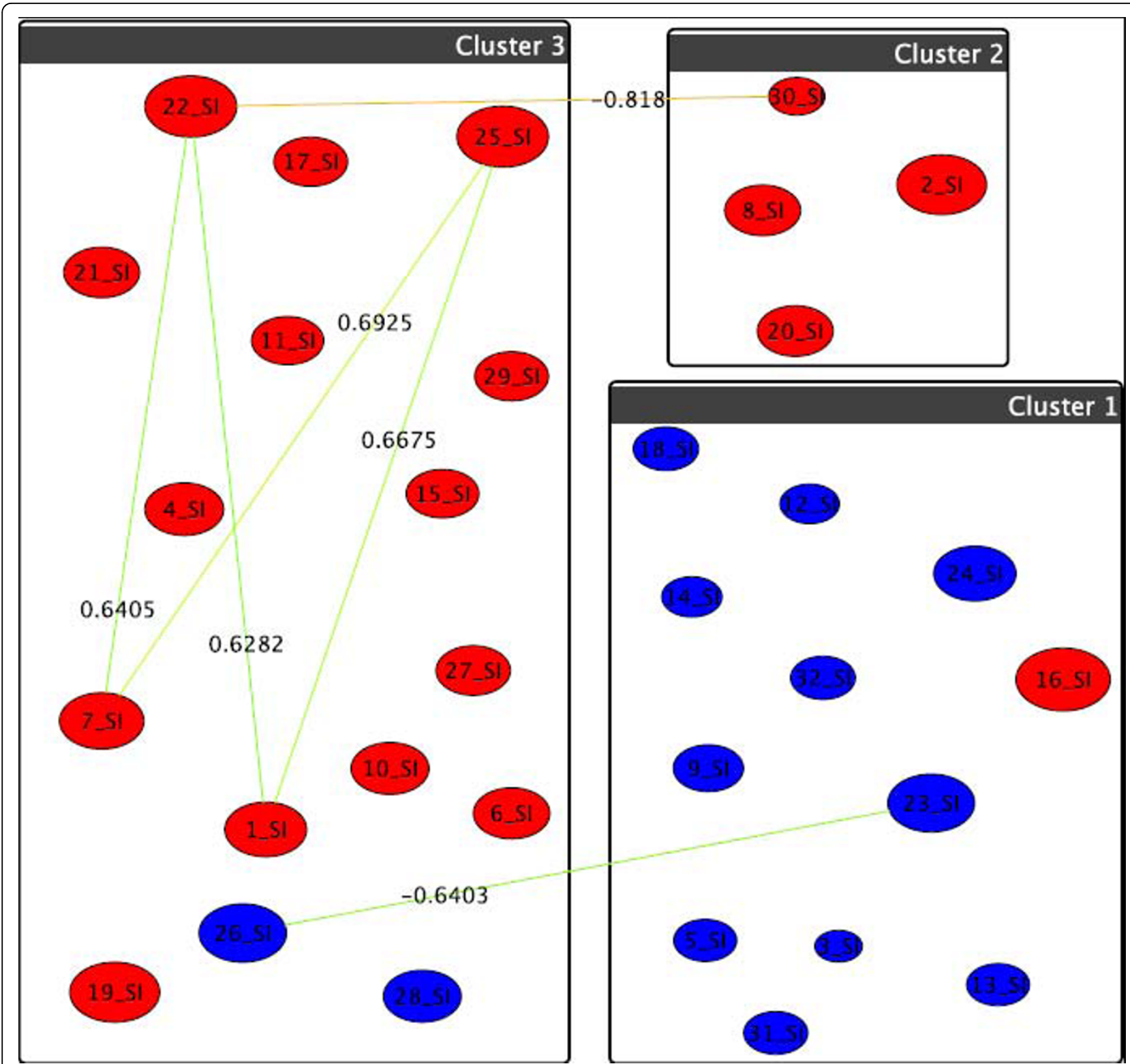

Figure 6 Graph-clustering correlation network for gangliosides showing the difference between U87+SN38/24hr+DI312/24hr and U87 +SN38/24hr+p53/24hr. Correlation network resulting from graph-clustering data analysis for a correlation threshold $>0.6$ for gangliosides showing no significant changes between the treatments U87+SN38/24hr+DI312/24hr (T3, boxes) versus U87+SN38/24hr+p53/24hr (T4, colors). Here, $v_{16}=$ GM2a (d18:1/24:0) leaves the box of blue nodes in T4, as T4 discriminates it from the blue nodes' values. Despite of low differences in correlation between T3 and T4, in T3 (boxes) there are three different clusters induced by groups of similarly behaving lipids, and only two for T4.]

with the PIs, no significant changes between the treatments U87+SN38/24hr+DI312/24hr versus U87+SN38/ $24 \mathrm{hr}+\mathrm{p} 53 / 24 \mathrm{hr}$ and $\mathrm{U} 87+\mathrm{DI} 312 / 24 \mathrm{hr}$ versus U87+p53/ $24 \mathrm{hr}$.

We want to emphasize - based on the achieved results - that graph clustering networks compared to traditional correlation networks represent a unique "fingerprint" in lipidomics by revealing analytic properties while displaying the graph structure. This term from the field of analytic visualizations signifies a superficial drawing on the one hand, as it masks or even ignores some aspects of the raw data, however, on the other hand, it implies that prominent and potentially unknown features of the data become visible in an easily recognizable way. The above investigations show how observations made with the help of our clusterings coincide with and corroborate findings and interpretations based on expert knowledge. Graph clustering and visualizations certainly cannot replace traditional analysis, but they can be a powerful supporting tool. 


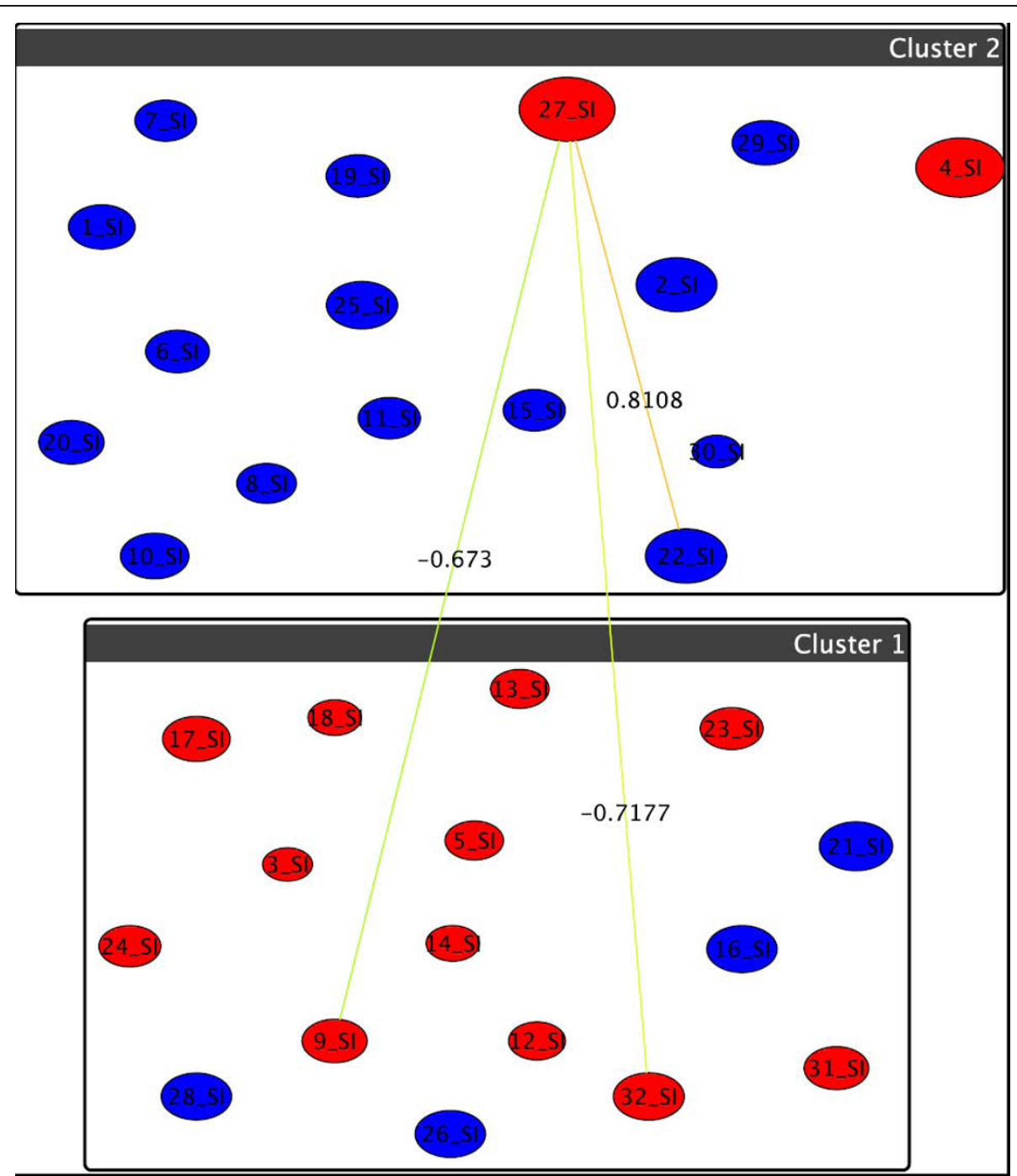

Figure $7 \mathrm{Graph}$-clustering correlation network for gangliosides showing the difference between U87+DI312/24hr and U87+p53/24hr Correlation network resulting from graph-clustering data analysis for a correlation threshold $>0.6$ for gangliosides showing no significant changes between the treatments U87+DI312/24hr (T5, boxes) versus U87+p53/24hr (T6, colors). Most notably, the size of $v_{27}=\mathrm{GD} 1$ (d18:1/16:0) and $v_{4}=\mathrm{GM} 3(\mathrm{~d} 18: 1 / 16: 0)+\mathrm{O}$ show their deviation from the behavior of the other nodes in the upper cluster, for T6, the two move to the red cluster. Most others do not di er heavily in their correlations.

\section{Conclusions}

In this paper, we analyzed and interpreted lipidomic data sets acquired by high-throughput measurements such as nano liquid chromatography (nano-LC) separation followed by detection with high mass accuracy and high mass-resolving power 14.5 FT-ICR MS. The samples were taken from U87 cell line treated under seven different treatments. We hypothesize that the observed correlations between the gangliosides concentrations are a result of the underlying lipidomic reaction network. Thus, lipidomic networks in glioblastoma represent highly dynamical processes continuously changing under the influence of fluctuations. These induce a specific pattern of correlations and are measured in the experiment as a result of network propagation.

As seen in plant metabolism [16], the determined pair-wise correlations represent a snapshot or "finger print" of the biochemical state of the glioblastoma cell at a given point in time. We introduced a novel concept in correlation network, the so-called graph clustering approach, which results in finding not only the correlations but also the clusters in these networks providing thus based on the concept of modularity a better visualization and analysis in lipidomic data exploration.

The simulations based on the novel theoretical concept have confirmed the experimental findings: a 


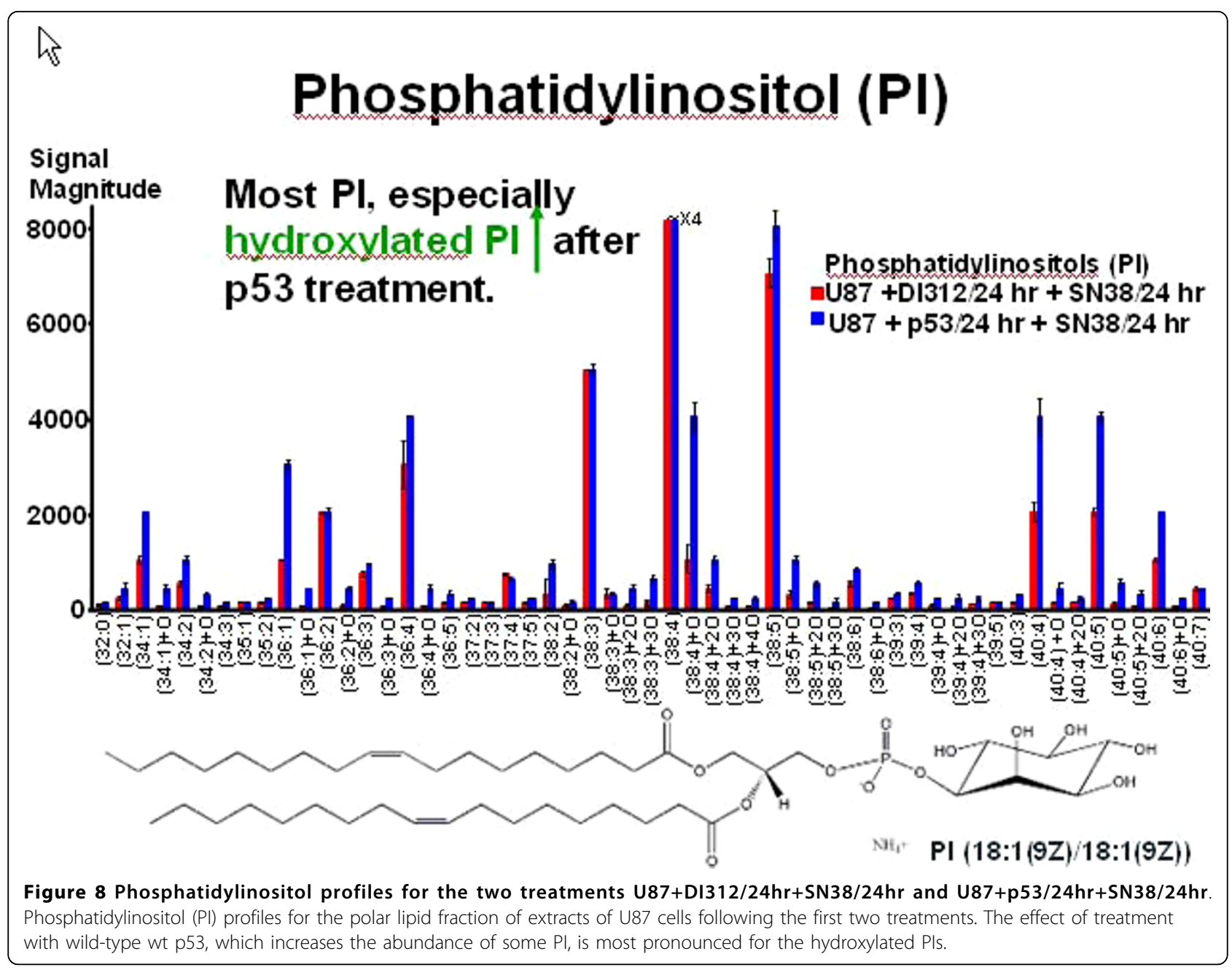

down-regulation for gangliosides and an up-regulation for sulfatides and phosphatidylinositol. Furthermore, we could quantize the regulations across the whole lipidom showing additionally that hydroxylated phosphatidylglycerol is also up-regulated. Among the seven possible treatments, we showed that most visible changes leading to apoptosis can be found in combination with adenovirus therapy.

In summary, this paper revealed in an unique way the "fingerprints" in lipidomics showing us how gene therapy (adeno virus) with wild type p53 followed by chemotherapy with the topoisomerase inhibitor SN38 induces 95\% apoptosis (cell death).

\section{Methods}

\section{Data Acquisition and Analysis}

The lipid analysis method has been previously described in [11], an abbreviated description of the method is outlined below.

\section{Cell culture}

The glioma cell line U87 MG (ATCC \#HTB-14) was grown in the presence of DMEM-F12 media supplemented with 10\% FBS (Cell Gro, Mediatech, Herndon, VA) in a humidified $\mathrm{CO}_{2}$ incubator at $5 \% \mathrm{CO}_{2}$. Cell cultures were grown in $150-\mathrm{mm}$ dishes to $90 \%$ confluency.

\section{Treatment of cells}

Cells $\left(\sim 2 \times 10^{6}\right)$ were treated with adenoviruses (therapeutic Ad-p53 or DI312 control adenovirus vector) or cytotoxic chemotherapy (SN-38), either alone, in combination or in different sequences. Cell cultures were treated for 24 h with SN-38 at a final concentration of 0.1 $\mu \mathrm{M}$ (stock solution of $10 \mathrm{mM}$ ).

Cell cultures were also treated with either control virus DI312 or test virus that contained wild-type p53 gene inserted in the $\mathrm{E} 1$ region of the adenovirus vector (Ad-p53) at 1:100 MOI (multiplicity of infection) from a stock virus titered at $2.8 \times 10^{11} \mathrm{pfu}$ (plaque-forming units). Cell cultures that were treated with a 


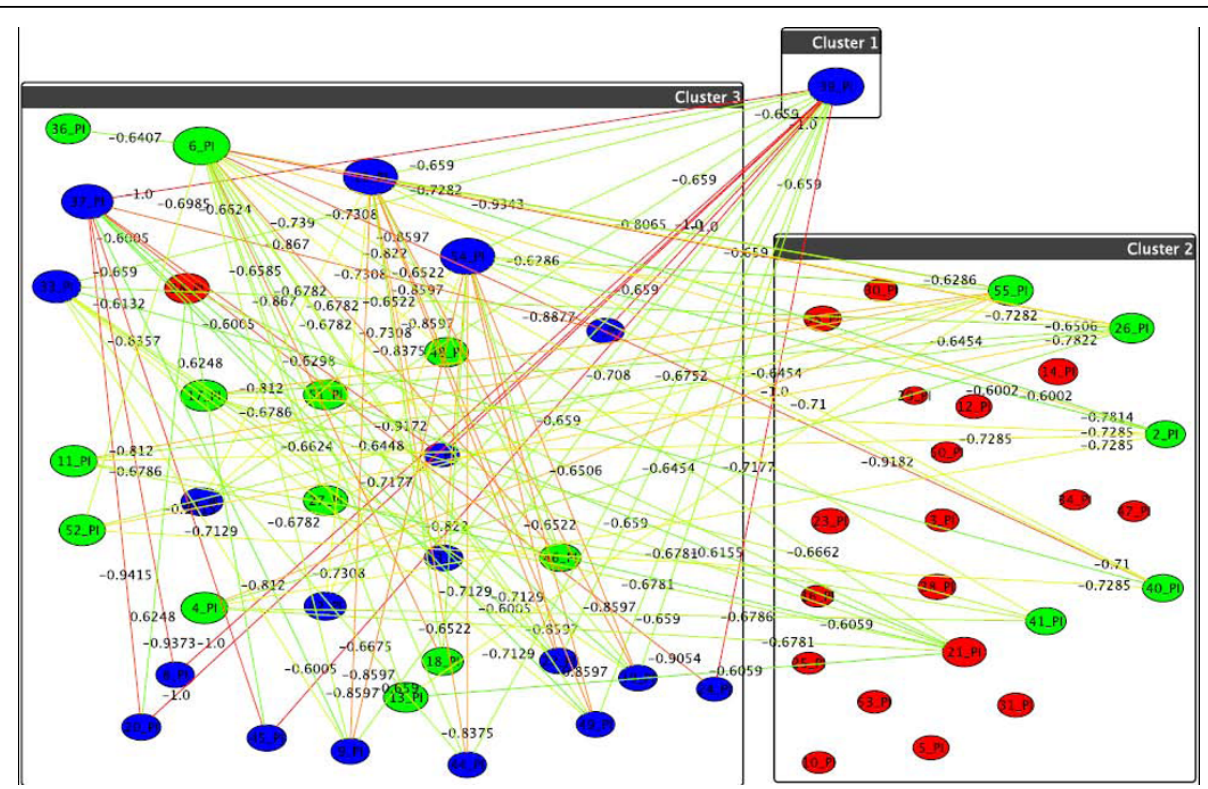

Figure 9 Graph-clustering correlation network for phosphatidylinositol showing the difference between U87+DI312/24hr+SN38/24hr and U87+p53/24hr+SN38/24hr. Correlation network resulting from graph-clustering data analysis for a correlation threshold $>0.6$ for Pls showing the difference between the first two treatments. Despite of the visual clutter, we here retain threshold for the sake of comparability to the above figures. Quite obviously, T1 and T2 heavily differ, with almost 7\% of all pairwise differences above 0.6. Red nodes are mostly small, which indicates little discrimination between $\mathrm{T} 1$ and $\mathrm{T} 2$ for these nodes, which in the raw data correspond to lipids with high values. Quite noticeable, $v_{39}=(38: 6)+\mathrm{O}$ shows the highest increase in $\mathrm{T} 2$ simply because its value is 0 in $\mathrm{T} 1$ but 100 in $\mathrm{T} 2$ while $v_{6}=(34: 2)+\mathrm{O}, v_{15}=(36: 3)+\mathrm{O}$, $v_{37}=(38: 5)+30$ and $v_{54}=(40: 6)+O$ are also significant.

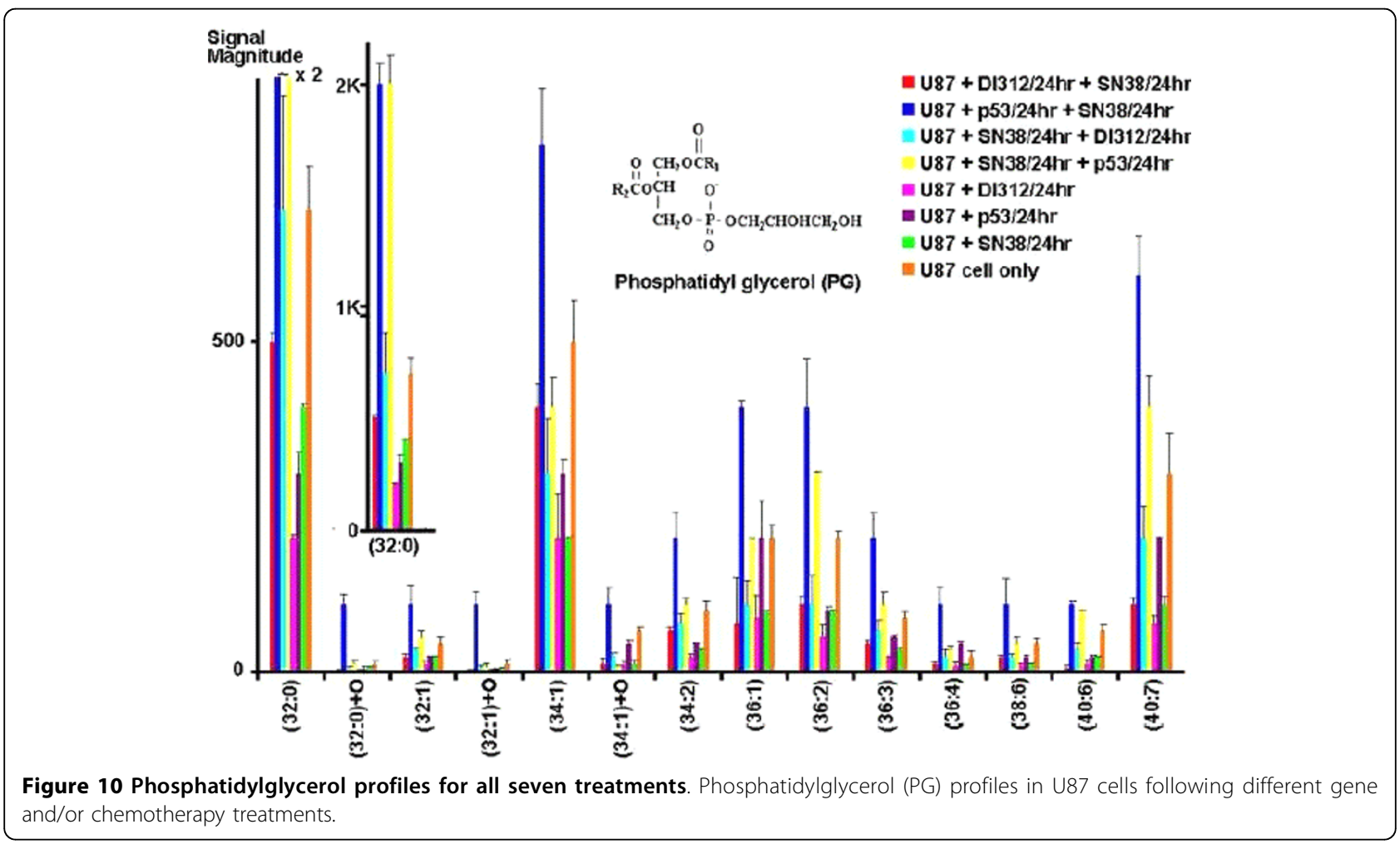




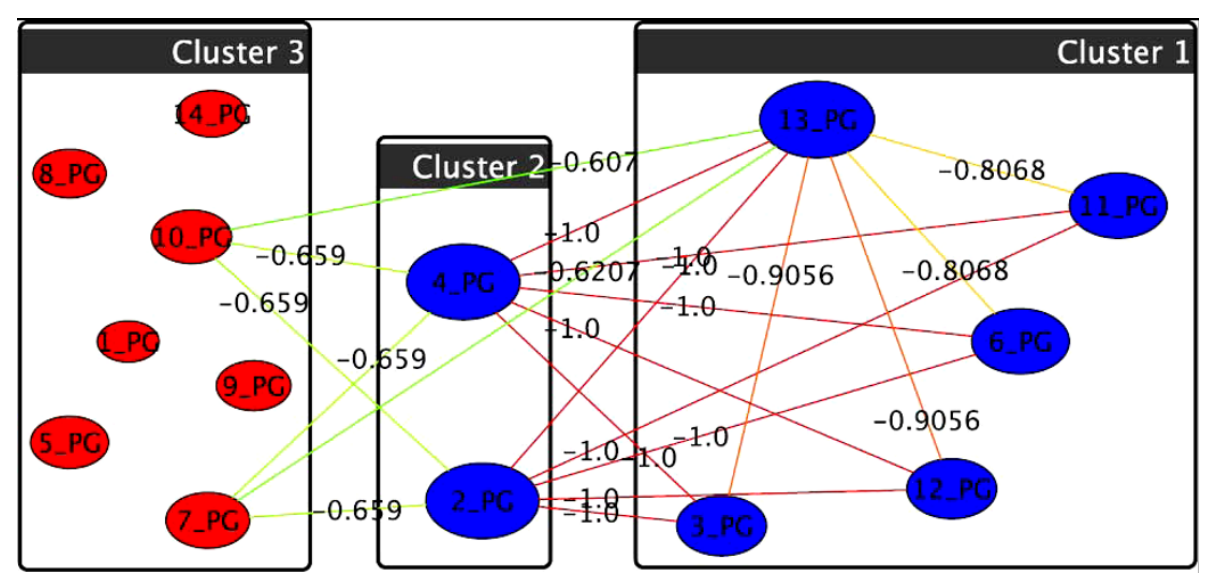

Figure $11 \mathrm{Graph}$-clustering correlation network for phosphatidylglycerol showing the difference between U87+DI312/24hr+SN38/24hr and U87+p53/24hr+SN38/24hr. Correlation network with threshold 0.6 for phosphatidylglycerols for $\mathrm{T} 1$ and $\mathrm{T} 2$. Very clearly $v_{2}=(32: 0)+0$ and $v_{4}=(32: 1)+0$ stand out. in T2 (colors) they abruptly behave as Cluster $1, v_{13}=(40: 6)$ parallels this, but much weaker. They went from close to 0 for T1 to 100 in T2. Since the left hand nodes retain their cluster they hardly differ in T1 and T2.

combination of drug and virus included a total incubation period of $48 \mathrm{~h}$ allowing $24 \mathrm{~h}$ for each agent. Cells were washed three times with room-temperature phosphate-buffered saline (PBS) between treatments. Prior to viral infection, the cells were placed in serum-free media for 1 hour to ensure adequate absorption of virus to the cells. These different conditions are listed in Table 1.

\section{Polar lipid extraction}

Cells (ca. $2 \times 10^{6}$ ) were extracted by the addition of methanol:chloroform 1:1 and sonicated for $30 \mathrm{~min}$. The extract was incubated overnight at $48^{\circ} \mathrm{C}$ in order to optimize GSL yields. After centrifugation, the supernatant was collected and partitioned with additional chloroform and $\mathrm{H}_{2} \mathrm{O}$. The upper layer was collected and dried. Approximately $\frac{1}{50}$ th of the total extract was consumed per LC/MS experiment.

\section{$n L C / M S$}

The lipids were reconstituted in $80 \%$ methanol (aq) with the addition of $10 \mathrm{mM} \mathrm{NH}_{4} \mathrm{OAc}$ and separated by nanoliquid chromatography (Eksigent, Dublin, CA) in a selfpacked $80 \mathrm{~mm} \times 50$ micron phenyl-hexyl column. The gradient was $\frac{15 \%}{85 \%}$ to $\frac{2 \%}{98 \%} \frac{A}{B}$ during 4 min (Solvent A. 98\% $\mathrm{H}_{2} \mathrm{O}, 2 \%$ methanol, and $10 \mathrm{mM} \mathrm{NH}_{4} \mathrm{OAc}$; B: 98\% methanol, $2 \% \mathrm{H}_{2} \mathrm{O}, 10 \mathrm{mM} \mathrm{NH}_{4} \mathrm{OAc}$ ) with a flow rate of $400 \mathrm{~nL} / \mathrm{min}$. LC effluent was analyzed on-line by negativeion micro-electrospray and a modified hybrid linear ion trap FT-ICR MS equipped with a 14.5 T magnet [12]. Parent ion mass spectra were collected at high mass resolving power $\left(m / \Delta m_{50 \%}=200,000\right.$ at $m / z 400$, in which $\Delta m_{50 \%}$ is spectral peak full width at half-maximum peak height) and acquisition rate $(>1 \mathrm{~Hz})$. The instrument was calibrated with a mixture (ESI calibration solution, Thermo Fisher, Waltham, MA) based on the quadrupolar trapping potential approximation [30,31]. Typical broadband external calibration mass accuracy is better than $500 \mathrm{ppb}$. Datadependent MS/MS was performed in the linear ion trap (CID, collisional induced dissociation) during collection of the ICR time-domain data [11]. Lipids were identified based on accurate mass and CID MS/MS tandem mass spectra in the LTQ. We compared tandem mass spectra to those in the literature as well as collected tandem mass spectra of lipid standards.

\section{Comparative Lipid Quantitation}

Comparative lipid quantitation was performed by a Semi-quantitative method based on the relative ion signal magnitude detected in FT-ICR MS of specific lipid species (glycolipid and phospholipid) for control vs. treated samples (transfectants). FT-ICR mass spectral signal magnitude is directly proportional to the number of ions of that mass-to-charge ratio [32]. Lipids were extracted, separated and analyzed under identical conditions. The complexity of the spectra per scan is simplified after nLC separation. The lipid species were well separated based on their polar head groups as well as ceramide or acyl backbones. Thus the competition for charge in the electrospray process is minimized as well as dynamic range issues in detection.

\section{Preprocessing}

Although the measurement domain already is $\mathcal{R}_{+}^{\prime}$, the data has a strongly skew distribution, such that any linear scaling function lets very large values overshadow important distinctions among smaller values. For two measurements $m_{u}$ and $m_{v}$ we thus use the logarithm inside the common correlation index $\operatorname{cor}\left(m_{u}, m_{v}\right)=\frac{\log \left(1+\min \left\{m_{u}, m_{v}\right\}\right)}{\log \left(1+\max \left\{m_{u}, m_{v}\right\}\right)} \quad$ (we define $\operatorname{cor}(0,0)$ 
$:=0)$ which is a symmetric two-variate function with codomain $[0,1]$. However, preliminary models suggested this function to be too non-disciminative; we found that a cubic exponent yielding $\omega(u, v)=\left(\operatorname{cor}\left(m_{u}, m_{v}\right)\right)^{3}$ enhances the resolution, with values well spread out in the interval $[0,1]$. Note that $\omega$ can easily incorporate any additional data on the relationship of lipids, e.g., measurements on lipid signaling.

\section{Acknowledgements}

This work was supported by DFG (German Research Foundation, Project SPP1307, Grant Number WA 654/15-2), NSF Division of Materials Research through DMR-06-54118, the State of Florida, the CERN Foundation, Dr. Marnie Rose Foundation, and John C. Merchant Memorial Fund.

\section{Author details}

${ }^{1}$ Department of Computer Science, Karlsruhe Institute of Technology, Karlsruhe D-76128, Germany. ${ }^{2}$ Department of Scientific Computing, Florida State University, Tallahassee, Florida 32310-4120, USA. ${ }^{3}$ Ion Cyclotron Resonance Program, National High Magnetic Field Laboratory, Florida State University, Tallahassee, Florida 32310-4005, USA. ${ }^{4}$ Department of Chemistry and Biochemistry, Florida State University, Tallahassee, Florida 32306, USA. ${ }^{5}$ The University of Texas M. D. Anderson Cancer Center, Houston, TX 77030, USA.

\section{Authors' contributions}

RG developed the graph clustering methodology, performed data analysis and drafted the manuscript. AMB developed the lipid informatics methodology and drafted the manuscript. DW developed the graph clustering methodology. $\mathrm{HH}$ and MRE performed the experiments with $\mathrm{nLC} /$ MS and drafted the manuscript. CAC initiated the study and performed data analysis. All authors read and approved the final manuscript.

Received: 15 March 2010 Accepted: 7 September 2010

Published: 7 September 2010

\section{References}

1. Chuang S: Mathematic models for cancer chemotherapy: pharmacokynetic and cell kinetic considerations. Cancer Chemotherap Rep 1975, 59:827-842.

2. Swanson K, Alvord E, Murray J: Dynamics of a model for brain tumors reveals a small window for the therapeutic intervention. Discrete Continuous Dynamical Systems 2004, 4:289-295.

3. Tracqui $P$, Mendjeli M: Modelling three-dimensional growth of brain tumors from time series of scans. Mathematical Models and Methods in Applied Sciences 1999, 9:581-598.

4. Stamatakos G, Antipas V, Uzunoglu N: A spatiotemporal, patient individualized simulation model of solid tumor response to chemotherapy in vivo: the paradigm of glioblastoma multiforme treated by temozolomide. IEEE Transactions on Biomedical Engineering 2006, 53:1467-1477.

5. Cerrato JA, Yung WK, Liu TJ: Introduction of mutant p53 into a wild-type p53-expressing glioma cell line confers sensitivity to Ad-p53-induced apoptosis. Neurooncology 2001, 3:113-122.

6. Puchades M, Nilsson C, Emmett M, Aldape K, Ji Y, Lang F, Liu T, Conrad C: Proteomic Investigation of Glioblastoma Cell Lines Treated with WildType p53 and Cytotoxic Chemotherapy Demonstrates an Association between Galectin-1 and p-53 Expression. Journal of Proteome Research 2007, 6:869-875.

7. Nielsen L, Maneval D: p53 Tumor Supressor Gene Therapy for Cancer. Cancer Gene Therapy 1998, 5:52-63.

8. Lang F, Shono T, Gilbert M: Ad-p53 sensitizes wild-type p53 gliomas to the topoisomerase I inhibitor SN-38. Neuro-Oncol 2002, 4:323-324.
9. Kopitz J, von Reitzenstein C, Burchert M, Cantz M, Gabius HJ: Galectin-1 Is a Major Receptor for Ganglioside GM1, a Product ofthe Growth-controlling Activity of a Cell Surface Ganglioside Sialidase, on Human Neuroblastoma Cells in Culture. J Biol Chem 1998, 273:11205-11211.

10. Segui B, Andrieu-Abadie N, Jaffrezou JP, Benoist $H$, Levade T: Sphingolipids as modulators of cancer cell death: Potential therapeutic targets. Biochim Biophys Acta 2006, 1758:8423-8430.

11. He H, Conrad C, Nilsson C, Emmmett M, Ji Y, Lang F, Marshall A, Schaub T: A novel method for lipidomic analysis: p53 expression modulates sulfatide, ganglioside and phospholipid composition at U87 MG glioblastoma cells. Anal Chem 2007, 79:8423-8430.

12. Schaub T, Blakney G, Hendrickson C, Quinn J, Senko M, Marshall A: High Performance Mass Spectrometry: Fourier Transform Ion Cyclotron Resonance at 14.5 Tesla. Anal Chem 2008, 80:3985-3990.

13. Gopalacharyulu P, Lindfors E, Miettinen J, Bounsaythip C, Oresic M: An Integrative Approach for Biological Data Mining and Visualization. Int J Data Mining and Bioinformatics 2008, 2:54-77.

14. Yetukuri L, Katajamaa M, Medina-Gomez G, Seppanen-Laakso T, VidalPuig A, Oresic M: Bioinformatics Strategies for lipidomic Analysis: Characterization of Obesity Related Hepatic Steatosis. BMC Systems Biology 2007, 1:1-15.

15. Oresic M: Bioinformatics and Computational Approaches Applicable to Lipidomics. Eur J Lipid Sci Technol 2009, 111:99-106.

16. Steuer R, Kurths J, Fiehn O, Weckwerth W: Observing and Interpreting Correlations in Metabolomic Networks. Bioinformatics 2003, 19:1019-1026.

17. Steuer R: On the Analysis and Interpretation of Correlations in Metabolomic Data. Briefings in Bioinformatics 2006, 7:151-158

18. Brandes U, Erlebach T, Eds: Network Analysis: Methodological Foundations, Lecture Notes in Computer Science Springer 2005, 3418[http://springerlink. metapress.com/content/nv20c2jfpf28/].

19. Meyers RA, Ed: Encyclopedia of Complexity and Systems Science Springer 2009 [http://www.springer.com/physics/book/978-0-387-75888-6].

20. Newman MEJ, Girvan M: Finding and evaluating community structure in networks. Physical Review E 2004, 69:(026113)[http://link.aps.org/abstract/ PRE/v69/e026113].

21. Brandes U, Delling D, Gaertler M, Görke R, Höfer M, Nikoloski Z, Wagner D: On Modularity Clustering. IEEE Transactions on Knowledge and Data Engineering 2008, 20(2):172-188[http://doi.ieeecomputersociety.org/10.1109/ TKDE.2007.190689].

22. Fortunato S: Community detection in graphs. Physics Reports 2009, 486(35):75-174[http://www.sciencedirect.com/science/journal/03701573].

23. Görke R: An Algorithmic Walk from Static to Dynamic Graph Clustering. PhD thesis Fakultät für Informatik 2010 [http://digbib.ubka.uni-karlsruhe.de/ volltexte/1000018288].

24. Blondel V, Guillaume UL, Lambiotte R, Lefebvre E: Fast unfolding of communities in large networks. Journal of Statistical Mechanics: Theory and Experiment 2008, 2008(10).

25. Delling D, Görke R, Schulz C, Wagner D: ORCA Reduction and ContrAction Graph Clustering. In Proceedings of the 5th International Conference on Algorithmic Aspects in Information and Management (AAIM'O9), Lecture Notes in Computer Science. Edited by: Goldberg AV, Zhou Y. Springer; 2009:5564:152-165.

26. Flake GW, Tarjan RE, Tsioutsiouliklis K: Graph Clustering and Minimum Cut Trees. Internet Mathematics 2004, 1(4):385-408[http://www. internetmathematics.org/volumes/1.htm].

27. Brandes U, Gaertler M, Wagner D: Engineering Graph Clustering: Models and Experimental Evaluation. ACM Journal of Experimental Algorithmics 2007, 12(1.1):1-26[http://portal.acm.org/citation.cfm?id=1227161.1227162].

28. Delling D, Gaertler M, Görke R, Wagner D: Engineering Comparators for Graph Clusterings. Proceedings of the 4th International Conference on Algorithmic Aspects in Information and Management (AAIM'08), Lecture Notes in Computer Science Springer 2008, 5034:131-142.

29. Rand WM: Objective Criteria for the Evaluation of Clustering Methods. Journal of the American Statistical Association 1971, 66(336):846-850[http:// www.jstor.org/stable/2284239? origin=crossref].

30. Ledford E, Rempel D, Gross M: Space Charge Effects in Fourier Mass Spectrometry - Mass Calibration. Analytical Chemistry 1984, 56:2744-2748. 
31. Shi S, Drader J, Freitas M, Hendrickson C, Marshall A: Comparison and Interconversion of the two most common frequency-to-mass calibration functions for Fourier transform ion cyclotron resonnace mass spectrometry. Journal of Mass Spectrometry 2000, 195/196:591-598.

32. Marshall A, Hendrickson C, Jackson G: Fourier Transform Ion Cyclotron Resonance Mass Spectroscopy: A Primer. Mass Spectrometry Reviews 1998, 17:1-35

doi:10.1186/1752-0509-4-126

Cite this article as: Görke et al:. Determining and interpreting

correlations in lipidomic networks found in glioblastoma cells. BMC Systems Biology 2010 4:126.

Submit your next manuscript to BioMed Central and take full advantage of:

- Convenient online submission

- Thorough peer review

- No space constraints or color figure charges

- Immediate publication on acceptance

- Inclusion in PubMed, CAS, Scopus and Google Scholar

- Research which is freely available for redistribution

Submit your manuscript at www.biomedcentral.com/submit
C Biomed Central 